\title{
Choi Response Criteria in the Evaluation of Gastrointestinal Stromal Tumors
}

National Cancer Institute

\section{Source}

National Cancer Institute. Choi Response Criteria in the Evaluation of Gastrointestinal

Stromal Tumors. NCI Thesaurus. Code C122394.

A composite set of criteria developed by Choi et al. using imaging techniques in the

evaluation of gastrointestinal stromal tumors response to imatinib mesylate treatment.

These criteria integrate changes in tumor size and tumor density. 